\title{
Arterial Hypertension-A Disease of the Juxtaglomerular Apparatus?
}

\author{
A. Erik G. Persson and Ulf Boberg \\ Department of Urology, University Hospital, and the Department of Physiology \\ and Medical Biophysics, University of Uppsala, Sweden
}

\begin{abstract}
From a special strain of genetically hypertensive rats, the Milan hypertensive strain (MHS), arterial hypertension can be transplanted with the kidney to the Milan normotensive strain (MNS). During development of hypertension in MHS rats there was an activation of the tubuloglomerular feedback control that reduced glomerular filtration rate, leading to retention of electrolytes and fluid. This increased extracellular fluid volume reduces feedback sensitivity, but in a fashion that gives rise to chronic extracellular fluid expansion and can thereby raise the blood pressure. In a limited sense, arterial hypertension in these animals exists to prevent the kidney from retaining more extracellular fluid volume. The altered function in the juxtaglomerular apparatus of the MHS rats thus may explain the rise in arterial blood pressure.
\end{abstract}

\section{INTRODUCTION}

The tubuloglomerular feedback control (TCF) is important for the control of extracellular fluid volume and arterial blood pressure $(11,19)$. Recent experiments in our laboratory $(5,12,13)$ have indicated that malfunctioning of this mechanism may be the cause of development of arterial hypertension in rats of the Milan hypertensive strain (MHS). Some evidence in support of this concept is discussed in the present paper.

In recent years the great importance of the interstitial space and its pressures for the function of the kidney has become apparent. In the rat, the renal interstitial hydrostatic pressure $\left(P_{\text {int }}\right)$ is about $1-2 \mathrm{~mm} \mathrm{Hg}$ under control conditions, and increases by about $2 \mathrm{~mm} \mathrm{Hg}$ during saline volume expansion by $5 \%$ of body weight $(16,20)$. The interstitial oncotic pressure $(\pi$ int $)$ was found to be about $4-5 \mathrm{~mm} \mathrm{Hg}$ and to decrease by about $2 \mathrm{~mm} \mathrm{Hg}$ during saline volume expansion (16). Considerable change evidently occurred in the net interstitial pressure ( $\mathrm{P}_{\text {int }}-\boldsymbol{\pi}_{\text {int }}$ ) during saline volume expansion, a situation which we have 
described as "interstitial oedema". Under conditions of dehydration, on the other hand, the rats showed very low hydrostatic and high interstitial oncotic pressure, collectively designated "interstitial dehydration" in our studies.

In the discussion of volume regulation, the nephron may be considered as two units. One is volume-regulating, consisting of the glomerulus, proximal tubule, and loop of Henle to the macula densa, at which point the possibility of a feedback loop exists. The other unit may be called the fine-adjusting electrolyte and water excretory unit, consisting of the distal tubule and collecting ducts.

Concerning the volume-regulating unit, which we shall discuss in this paper, a large fluid volume is filtered into Bowman's space and from there flows into the proximal tubule. Fluid reabsorption in this segment usually is a constant fraction of the total filtrate, about $2 / 3$. It would seem that the mechanism of this fluid reabsorption is multifactorial, and also that a change in interstitial pressure induced by altered capillary pressure can modulate fluid transfer $(6,7)$. In a situation that leads to "interstitial oedema", the proximal tubular fluid reabsorption is reduced, whereas during "interstitial dehydration" it is increased. This alteration in fluid absorption rate may be due to an effect on a direct pressure-dependent fluid transfer across the proximal tubular wall (1, 10). The next segment down the nephron through which the fluid passes is the loop of Henle. During this passage electrolytes are preferentially reabsorbed, while water is transported to a lesser extent. This explains the low sodium concentration in the early distal tubule. If the fluid flow through this segment is increased, electrolyte reabsorption will also be increased, but not fully proportionate to the increase in flow, with the result that the early distal sodium chloride concentration, which normally is low, will rise $(8,14)$.

The site of contact between the altered cells in the distal tubule, the macula densa cells and arterioles in the vascular pole of the kidney, and the renin-granule-containing cells constitute the juxtaglomerular apparatus. This is an important regulatory unit, that can sense the chloride concentration in the distal tubular fluid. The system is activated when the electrolyte concentration increases, leading to reduction in glomerular capillary pressure and glomerular filtration rate (GFR) via activation of the TGF mechanism, and to increased renin release. The basic function of a volume-regulating unit in the nephron can now be outlined.

If GFR for some reason tends to increase as a result of constant fractional proximal reabsorption, the fluid delivery to the loop of Henle will be enhanced. An increased flow through this nephron segment will increase the electrolyte concentration in the early distal tubule and thereby activate the juxtaglomerular apparatus to lower the GFR and produce renin. It has also been found, however, that the TGF mechanism is only slightly activated under control 
conditions $(11,15)$, i.e. the operational point is close to no activation at all (Fig. 1). This means that under control conditions there is very little activation of the TGF system, just as there is only very low renin production.

The question therefore arises: When is the feedback system activated to effect appreciable reduction of GFR ? From several lines of evidence we have deduced that changes in the extracellular fluid volume of the blood pressure will alter the sensitivity of the TGF via changes in renal interstitial hydrostatic and oncotic pressures (11). Under conditions of saline volume expansion or postunilateral nephrectomy, or in the initial period of ureteral occlusion - all situations with "interstitial oedema" - a low or abolished TGF response was found, as shown by a shift of the response curve to the right in Fig. 1. This of course constitutes an important resetting of the sensitivity to avoid reduction of GFR in a situation with demand for increased fluid excretion. GFR consequently can remain high despite increased distal delivery of fluid. In situations such as saline volume expansion, renin release also is low.

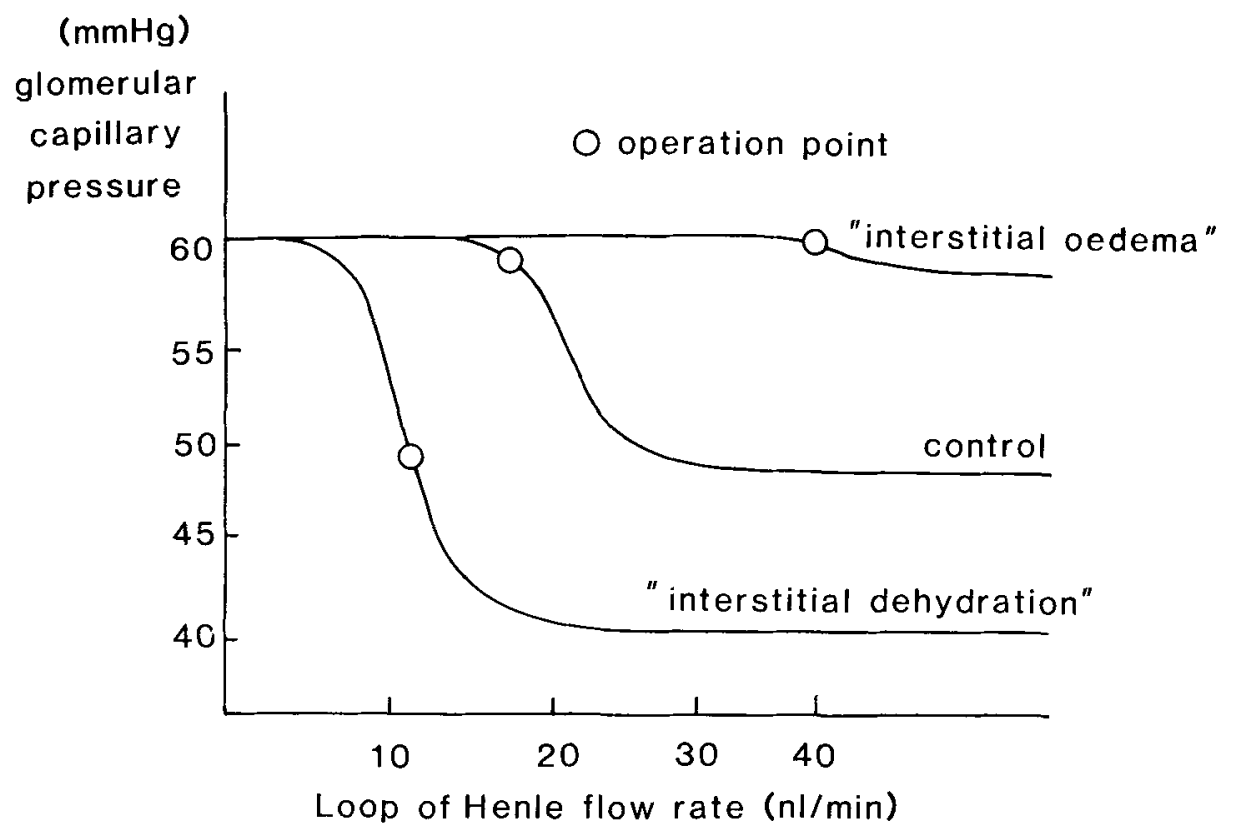

Fig. 1. Glomerular capillary pressure estimated for stop-flow pressure in relation to the flow rate in the loop of Henle. Comparisons under control conditions or with "interstitial oedema" or "interstitial dehydration". The operation point for the mechanism is shown. 
In states of dehydration, hypovolaemia or hypotension, or following unilateral ureteral occlusion - all situations with "interstitial dehydration" - we found high TGF sensitivity with a low flow threshold and a large reduction of, maximal glomerular capillary pressure in response to increased flow. This implies a shift of the response curve to the left compared with the control curve in Fig. 1. Further, in all of these situations with "interstitial dehydration", although the distal delivery of fluid was normal or even subnormal, the increase in sensitivity was so large that the feedback was greatly activated to reduce glomerular capillary pressure and GFR. In dehydration, hypovolaemia and hypotension the renin release increased, indicating that activation of the TGF mechanism also proceeds parallel with renin release, a concept which accords with findings in earlier direct micropuncture studies (9). The TGF thus is activated as a consequence of the extracellular fluid needs and the blood pressure level, and not solely as a result of increased delivery of fluid.

This interrelationship, representing the influence of renal interstitial pressure on renal function, is illustrated in Fig. 2. GFR and tubular fluid reabsorption, the latter being influenced by interstitial pressure, determine the excretion rate. Furthermore, GFR and fluid reabsorption up to the early distal tubule determine the fluid load to the juxtaglomerular apparatus. The response of this apparatus then depends on the degree of stimulation of the renal interstitial pressure. In situations with high-degree stimulation of the interstitial pressure receptor mechanism, such as hypotension, hypovolaemia and dehydration, the sensitivity of the TGF mechanism will be so high that even normal or reduced distal delivery of fluid will be sufficient to activate the juxtaglomerular apparatus that reduces GFR and increases renin production. These alterations will in turn change the urine excretion rate, which will lead to restoration of the fluid balance towards the control level. This feedback control loop will then act to restore the disturbed extracellular fluid volume balance to the control level and to the control operation point. Such resetting of the TGF sensitivity is therefore an important determinant of body fluid volume and an important factor in normalisation of different types of volume or blood pressure disturbance. The TGF mechanism will strive to restore the fluid volume of the body so that it attains the operation point on the control curve. In this fashion the TGF can determine the total volume of body fluid.

The link between extracellular fluid volume and blood pressure is not yet clear, though theories concerning the mode of control have been proposed (17). It is clear, however, that in patients without kidneys the extracellular volume influences blood pressure. Since the TGF mechanism and its resetting are important for the actual volume of the extracellular fluid, we made comparisons of the TGF and its resetting between rats of the Milan hypertensive strain 


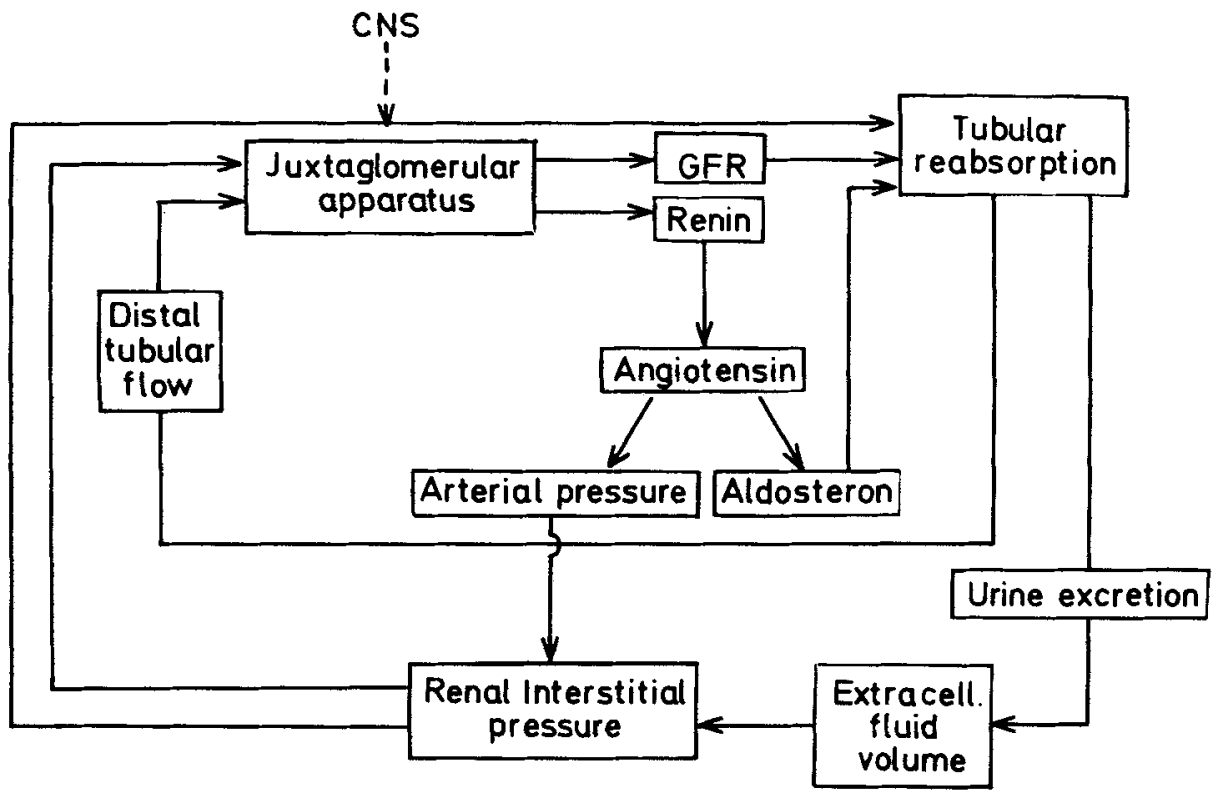

Fig. 2. Block diagram illustrating the influence of renal interstitial pressure in renal volume regulation.

Reproduced by permission from the Editor of Kidney International (from reference 11 ).

(MHS) and the Milan normotensive strain (MNS). These studies were of particular interest because the hypertensive disease in MHS rats can be transplanted with the kidney (2).

Because of differences in renal function, we studied MHS rats during three phases of life, and MNS rats at corresponding ages. The rats were first studied in the prehypertensive phase, after weaning at the age of 4-5 weeks, when their weight was $60-100 \mathrm{~kg}$. The second phase was during development of hypertension, which occurs at 5-7 weeks when the body weight is 90-150 g, and the third was an adult phase, at age about 3 months with weight 250-350 g. Earlier studies by ather authors (3) showed that MHS rats in the prehypertensive phase had high GFR and low renin production compared with MNS rats. In recent investigations $(5,12)$ we found that in the prehypertensive phase the MHS rats had no TGF response at all, while MNS rats showed normal response. This absence of a feedback response can explain the high GFR, since the operation point usually shows some slight activation of the TGF mechanism in response to reduced GFR. These findings are also in line with the low renin release that was observed.

During development of hypertension, by contrast, the MHS rats displayed a TGF mechanism with very high sensitivity, with a low flow threshold leading to 
activation of the TGF to reduce GFR. In this phase there is relative reduction in GFR and relative increase in renin production and sodium retention. These observations can readily be explained by the high feedback sensitivity, which will reduce GFR and retain electrolytes. The process thus will lead to fluid retention in the MHS animals that will tend to normalize TGF sensitivity but also increase arterial blood pressure. Indeed, in the adult MHS rats we found a normal TGF response. However, both in the adult MHS rats and during the development of hypertension, the resetting of TGF sensitivity was impaired. During volume expansion the high sensitivity was only slightly reduced, and was not reset to a lower level as it was in the MNS rats. We also found that the interstitial pressure was normal both under control conditions and during volume expansion. A change in interstitial pressure could therefore not explain the impaired TGF resetting.

The mechanism of action, speculatively, seems to be as follows. There is an increased transmembrane electrolyte transport in red cells and proximal tubular cells of MHS rats. Many cells in the MHS rat appear to have this transport abnormality (4). If the abnormal transport occurs also in the macula densa cells, an increase in chloride or sodium chloride transport may activate the feedback and explain the high sensitivity that develops in this situation. The impaired resetting during volume expansion will then keep GFR low and retain fluids and electrolytes even when there is a volume overload. The result will be a rise in arterial blood pressure. Both the volume expansion and the increase in blood pressure can then restore the TGF sensitivity to a normal level. Thus, in a limited sense it appears that the high arterial pressure in the MHS rats exists in order to prevent the kidney from retaining an even greater extracellular volume of fluid. These studies have convinced us that the TGF mechanism plays an important role in the development and maintenance of arterial hypertension in the MHS rats.

\section{REFERENCES}

1. Ågerup, B. \& Persson, A.E.G.: Modulation of proximal tubular hydraulic conductivity by peritubular capillary oncotic pressure. Acta Physiol Scand $115: 355-359,1982$.

2. Bianchi, G., Fox, U., Difrancesco, C.F., Giovanetti, A.M. \& Pagetti, D.: Blood pressure changes produced by kidney crosstransplantation between spontaneously hypertensive rats and normotensive rats. Clin Sci Mol Med 47:435-448,1974.

3. Bianchi, G., Baer, P.G., Fox, U., Duzzi, L., Pagetti, D. \& Giovanetti, D.M.: Changes in renin, water balance and sodium balance during development of high blood pressure in genetically hypertensive rats. Circ Res 37:153-161,1975.

4. Bianchi, G., Ferrari, P. \& Barber, B.R.: The Milan hypertensive strain. In Handbook of Hypertension, vol. 4. Experimental and genetic models of 
hypertension. Ed. de Jons. Elsevier Scient Publ 1984.

5. Boberg, U. \& Persson, A.E.G.: Increased tubuloglomerular feedback activity in Milan hypertensive rats. Am J Physiol: Submitted.

6. Brenner, B.M., Falchuk, K.H., Keimowitz, R.L. \& Berliner, R.W.: The relationship between capillary protein concentration and fluid reabsorption by the renal proximal tubule. J Clin Invest 48:1519-1531,1969.

7. Ichikawa, I., Hoyer, J.R., Seiler, M.W. \& Brenner,B.M.: Mechanism of glomerulotubular balance in the setting of heterogeneous glomerular injury. J Clin Invest 69:185-198,1982.

8. Morgan, T. \& Berliner, R.W.: A study by continuous microperfusion of water and electrolyte movements in the loop of Henle and distal tubule of the rat. Nephron 6:388-405,1969.

9. Morgan, T. \& Davis, J.M.: Renin secretion at the individual nephron level. Pflügers Arch 359:23-31,1975.

10. Persson, A.E.G., Schnermann,J., Ågerup, B. \& Eriksson, N-E.: The hydraulic conductivity of the rat proximal tubular wall. Determinations with colloidal solution. Pflügers Arch 360:25-44,1975.

11. Persson, A.E.G., Boberg, U., Hahne,B., Müller-Suur, R., Norlén, B-J. \& Selén, G. Interstitial pressure as a modulator of the tubuloglomerular feedback control. Kidney Int 22:122-128,1982.

12. Persson, A.E.G., Bianchi, G. \& Boberg, U.: Evidence of defective tubuloglomerular feedback control in rats of the Milan hypertensive strain (MHS). Acta Physiol Scand 122:217-219,1984.

13. Persson, A.E.G., Bianchi, G. \& Boberg, U.: Tubuloglomerular feedback in hypertensive rats of the Milan strain. Acta Physiol Scand 123:135-146,1985.

14. Schnermann, J.: Microperfusion study of single short loop of Henle in rat kidney. Pflügers Arch 300:255-282,1968.

15. Schnermann, J., Persson, A.E.G. \& Ågerup, B.: Tubuloglomerular feedback: nonlinear relation between glomerular hydrostatic pressure and loop of Henle perfusion. J Clin Invest 58:862-869,1973.

16. Selén,G. \& Persson,A.E.G.: Hydrostatic and oncotic pressures in the interstitium of dehydrated and volume-expanded rats. Acta Physiol Scand 1.17: $75-81,1983$.

17. De Wardener, H.E. \& MacGregor, G.A.: Dahl's hypothesis that a saluretic substance may be responsible for a sustained rise in arterial pressure: Its possible role in essential hypertension. Kidney Int 18:1-9,1980.

18. Wolgast,M., Persson,E., Schnermann,J., Ulfendahl,H. \& Wunderlich,P.: The colloid osmotic pressure of the subcapsular interstitial fluid of rat kidneys during hydropenia and volume expansion. Pflügers Arch 340:123-131, 1973.

19. Wright, F.S. \& Briggs, J.P.: Feedback control of glomerular blood flow, pressure and filtration rate. Physiol Rev 59:958-1006,1979.

20. Wunderlich,P., Persson,E., Schnermann, J., Ulfendahl,H. \& Wolgast, M.: Hydrostatic pressure in the subcapsular interstitial space of rat and dog kidneys. Pflügers Arch 328:307-319,1971.

Address for reprints

A. Erik G. Persson

Department of Urology

University Hospital

S-751 85 Uppsala

Sweden 